\title{
Application of Regional Arterial Infusion Chemotherapy in Advanced Gastric Cancer
}

\author{
Hao Xu, Weicheng Wang, Xiaoming Zhang, Yi Zhou, Jing Zheng, Cui Yang, Lin Yang* \\ Sichuan Key Laboratory of Medical Imaging, Department of Radiology, Affiliated Hospital of North Sichuan Medical College, \\ Nanchong, China \\ Email: *linyangmd@163.com
}

How to cite this paper: $\mathrm{Xu}, \mathrm{H}$., Wang, W.C., Zhang, X.M., Zhou, Y., Zheng, J., Yang, C. and Yang, L. (2018) Application of Regional Arterial Infusion Chemotherapy in Advanced Gastric Cancer. Journal of Cancer Therapy, 9, 118-129.

https://doi.org/10.4236/jct.2018.92013

Received: December 31, 2017

Accepted: February 8, 2018

Published: February 11, 2018

Copyright $\odot 2018$ by authors and Scientific Research Publishing Inc. This work is licensed under the Creative Commons Attribution International License (CC BY 4.0).

http://creativecommons.org/licenses/by/4.0/

\begin{abstract}
Gastric cancer ranks as the fifth most common malignant tumor worldwide and is the third most common cause of cancer-related death. For advanced gastric cancer (AGC), neoadjuvant chemotherapy (NAC) can reduce its stage, increase the rate of radical resection, improve response to treatment, reduce the risk of local recurrence and improve survival rate. Regional arterial infusion chemotherapy (RAIC) is a form of NAC that involves directly injecting chemotherapeutic drugs into the tumor site through the tumor-feeding artery. RAIC increases the local drug concentration around the tumor, thereby improving the therapeutic responses and reducing the adverse effects of the drugs. In recent years, RAIC has attracted increasing attention. This article summarizes the basic principles, procedure, chemotherapy regimens, adverse drug reactions and complications, clinical applications and response evaluation of RAIC in the treatment of AGC.
\end{abstract}

\section{Keywords}

Gastric Cancer, Neoadjuvant Chemotherapy, Regional Arterial Infusion Chemotherapy

\section{Introduction}

Gastric cancer ranks as the fifth most common malignant tumor worldwide and is the third most common cause of cancer-related death [1]. Currently, surgical resection is still considered the only cure for gastric cancer. However, the symptoms of gastric cancer are not obvious. Most gastric cancers are diagnosed in the late-stage, when surgical treatments are not available options. Neoadjuvant chemotherapy (NAC) improves the rate of radical resection and long-term survival 
by reducing tumor stage [2]. Regional arterial infusion chemotherapy (RAIC) is a method of injecting chemotherapeutic drugs directly into the tumor tissue through a tumor-feeding artery to increase the concentration of the chemotherapy drug at the tumor lesion. RAIC improves the curative effect and reduces the adverse effects of chemotherapy drugs. In recent years, RAIC has attracted increasing attention [3]-[9]. This article summarizes the basic principles, procedure, chemotherapy regimens, adverse drug reactions and complications, clinical applications and response evaluation of RAIC in the treatment of AGC.

\section{Basic Principles of RAIC}

The efficacy of chemotherapy drugs is not only related to their pharmacological effects and the sensitivity of the lesion to the drugs but also drug concentration and the time of drug contact with the lesion at a suitable concentration. In conventional systemic chemotherapy, a drug flows through the superior vena cava or inferior vena cava and refluxes to the right side of the heart. After pulmonary circulation, the drug is pumped out from the left heart and distributed to the body (including lesions of the target organ). In this process, the drug is diluted in the blood. The drug concentration at the target organ is the same as the drug concentration in the peripheral plasma. Distribution of the drug to the target organ drug mainly depends on the amount of blood flow passing through the target organ. A method to increase drug concentration at the target organ is to increase the drug dose. Drug dose and peripheral plasma concentrations are associated with toxic adverse effects of the drug.

RAIC involves directly delivering chemotherapy drug to the tumor through a tumor-feeding artery. The method not only increases the local concentration of the chemotherapy drug in the tumor but also extends the time of the drug contact with the lesions by various means. Therefore, compared with systemic chemotherapy through the peripheral vein, RAIC can improve the curative effect and can reduce the adverse effects of drugs [6] [10].

\section{RAIC Procedure}

The Seldinger technique is commonly used to puncture the right femoral artery. Anangiographic catheter is placed in the celiac artery for digital subtraction angiography. A tumor-feeding artery (left gastric artery, right gastric artery, left gastroepiploic artery, right gastroepiploic artery, or gastroduodenal artery) is identified. Then, the tumor-feeding artery is subject to ultra-selective intubation and angiography. Once a clear range of lesions, blood supply, and other conditions are determined, chemotherapy drugs are slowly injected (Figure 1).

Puncturing the femoral artery is a convenient operation with a high success rate. The method is mainly used for short-term RAIC. Puncturing the axillary and subclavian arteries requires a relatively more complex technique. Because these types of punctures affect the activities of the patients, they are mainly used for long-term, intermittent chemotherapy (implantable port system) to avoid repeated punctures [11] [12]. 


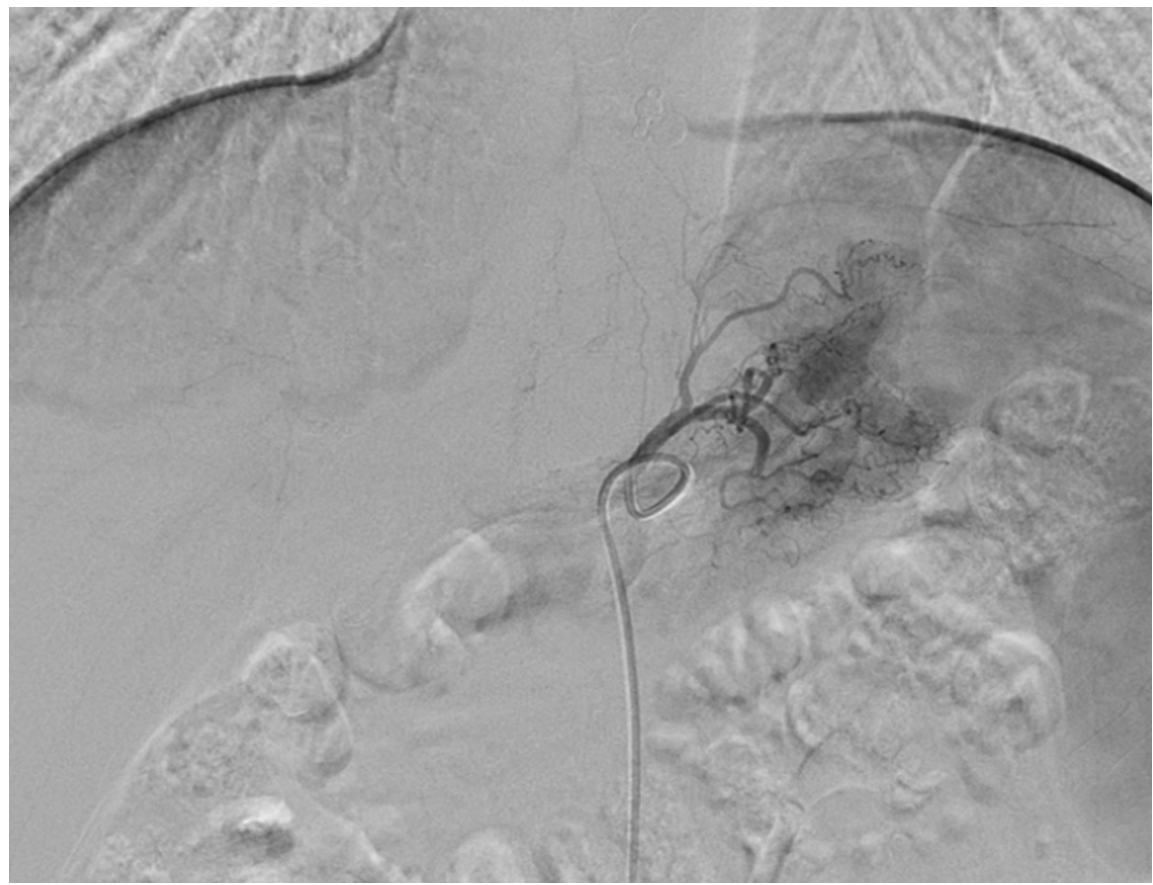

Figure 1. Thirty-year-old female patient with advanced gastric cancer. Digital subtraction angiography via the left gastric artery reveals the tumor vasculature.

\section{Chemotherapy Regimens}

When performing RAIC, physicians should choose concentration-dependent and tumor-sensitive drugs, use drugs of different mechanisms of action in combination, and avoid drugs with similar toxicity or cumulative toxicity to a same organ. Common clinical chemotherapy drugs include cisplatin, oxaliplatin, mitomycin, adriamycin, epirubicin, docetaxel, raltitrexed, and fluorouracil. There is currently no standard RAIC chemotherapy regimen, and physicians generally use drugs employed for systemic intravenous NAC. Commonly used chemotherapy regimens are shown in the following table (Table 1 ).

Treatment regimens vary from region to region. Among these regimens, those based on fluorouracil and platinum are very important [13] [14]. The dose of chemotherapeutic drug for RAIC is generally decided based on that of systemic chemotherapy and is adjusted according to the patient's body surface area and disease condition.

\section{Adverse Drug Reactions and Complications}

With the administration of RAIC, chemotherapy drugs will also reach the entire body though the blood circulation and act as systemic chemotherapy [3] [7] [9] [15]. The adverse drug reactions of RAIC are similar to those of systemic chemotherapy but to a lesser extent [7]. The major toxicity was bone marrow suppression; other side effects included appetite loss, nausea and vomiting [9]. Most bone marrow toxicities, appetite loss, nausea and vomiting were acceptable. Complications related to arterial puncture and intubation include hematoma at 
Table 1. Commonly used chemotherapy regimens.

\begin{tabular}{cc}
\hline Regimen & Drug composition \\
\hline FAM regimen & Fluorouracil, adriamycin, mitomycin \\
DCF regimen & Docetaxel, cisplatin, fluorouracil \\
ECF regimen & Epirubicin, cisplatin, fluorouracil \\
FCA regimen & Fluorouracil, cisplatin, adriamycin \\
FCM regimen & Fluorouracil, cisplatin, mitomycin \\
EOX regimen & Epirubicin, oxaliplatin, capecitabine \\
FOLFOX regimen & Folinic acid, fluorouracil, oxaliplatin \\
SOX regimen & S-1, oxaliplatin \\
\hline
\end{tabular}

the puncture site, arterial thrombosis, artery dissection, pseudoaneurysm, and arteriovenous fistula [16] [17] [18] [19]. Methods to avoid these complications include mastering the puncture technique, avoiding repeated punctures, choosing fine and soft guide wires and catheters, standardizing the intubation operation, and paying postoperative attention to oppression hemostasis. Hematoma at the site of femoral artery puncture represents the most common complication. Most of these hematomas can be self-absorbed. Pelvic hematoma caused by an overly high puncture point or retroperitoneal hematoma often results in a large amount of blood loss. Once diagnosed, the patient should immediately receive transfusion and anti-bleeding treatments, and if necessary, surgical repair. Femoral artery thrombosis is also a common complication of femoral artery puncture. Once diagnosed, the patient should immediately receive anticoagulation and thrombolytic treatments. Patients who have femoral artery pseudoaneurysm and arteriovenous fistula and do not effectively respond to compression method should receive prompt surgical repair.

As for any drug, iodine-based contrast agents can cause adverse events [20] [21] [22] [23]. Mild adverse effects of the contrasting agent include vomiting and rash. If necessary, the patient should receive injection of antihistamines or dexamethasone. Short-term symptoms are often reduced or disappear. Severe reactions manifest as dyspnea, tracheal spasm and shock. Patients should immediately receive adrenaline, oxygen inhalation and other treatments [20] [21] [22] [23]. For patients with impaired renal function, the physician should pay attention to contrast agent selection, dose control, and preoperative and postoperative hydration.

Vasovagal reflexes [24] manifest as a sudden drop in blood pressure, reduced heart rate, paleness, sweating, slow breathing, and disturbance of consciousness when the arterial sheath is removed. Once these symptoms occurred, emergency measures should be initiated, including maintaining the patient in the supine position, rapidly replenishing isotonic saline, and intravenous injection of atropine and dopamine. 


\section{Clinical Applications}

\subsection{Preoperative Chemotherapy}

The concept of NAC was proposed in 1982 [25]. Wilke et al. [15] reported the use of NAC in gastric cancer in 1989. In their study, when NAC was administered to 34 patients with unresectable AGCs, 33 patients exhibited reduced AGC stages and successfully underwent radical gastrectomy and lymph node clearance. The median survival time for all patients was 18 months, and the median disease-free survival time for all patients was 24 months. Subsequent studies have obtained similar results [4] [26] [27].

Given that neoadjuvant RAIC can improve the curative effect and reduce the adverse effects of chemotherapy drugs, it has received increasing attention in recent years. Studies have demonstrated that treating well defined AGCs with neoadjuvant RAIC can effectively reduce the AGC stage, improve the rate of radical resection, increase treatment response, and prolong survival [4] [28] [29] [30]. Tao et al. [29] studied the effects of preoperative RAIC in inducing apoptosis and inhibiting the growth of gastric cancer cells. A total of 110 cases of GC and 68 cases of metastatic lymph nodes with or without RAIC were adopted. The apoptotic index in the RAIC treatment group was significantly increased compared with the untreated group, whereas the proliferation index in the RAIC treatment group was significantly reduced compared with the untreated group. A postoperative survey demonstrated that the 5-year survival rate of GC patients in the RAIC group was significantly increased compared with the untreated group. Wu et al. [7] analyzed 178 AGCs receiving short-term neoadjuvant EOX chemotherapy. The postoperative pathological response rate was $46.1 \%$, and the complete remission rate was $2.2 \%$. Pathological response and tumor differentiation are significantly correlated with the modes of chemotherapy administration (intravenous and arterial regional perfusion chemotherapy). The 3-year overall survival (OS) and recurrence-free survival (RFS) rates were $67 \%$ and $53 \%$, respectively. Compared with the intravenous chemotherapy group, the RAIC group exhibited a better median RFS. In a study by He et al. [31], 47 AGC patients with para-aortic lymph node metastasis (PALM) received NAC. Forty-six patients completed 2 cycles of NAC. The total effective rate of NAC on primary tumors was $80.4 \%(37 / 46)$. The response rate of the para-aortic lymph node (PAL) was $76.1 \%$ (35/46). Thirty-two patients completed D2 radical surgery. Six patients achieved complete remission. The toxicity profile was well tolerable. No treatment-related deaths were noted. The median survival time of all patients was 23 months. The median survival time of patients in the nonsurgical and surgical groups were 12 months and 29 months, respectively. The 1-, 2-, and 3-year survival rates were $70.96 \%, 43.27 \%$, and $35.48 \%$, respectively, for all patients and $96.87 \%, 68.75 \%$, and $40.63 \%$, respectively, for patients in the surgical group.

However, reports with inconsistent findings are also noted in the literature [32] [33]. These findings suggest that NAC could reduce tumor stage and increase the rate of surgical R0 resections but has no clear advantage in improving 
long-term survival. Schuhmacher et al. [32] randomly divided 144 patients with AGC into two groups. Patients in one group received surgery after NAC, and patients in the other group exclusively underwent surgical resection. The R0 resection rate of the group that received surgery after NAC was $81.9 \%$, which was significantly increased compared with the $66.7 \%$ rate $(P=0.036)$ in the simple surgical resection group. No significant difference in postoperative complications was noted between the two groups $(27.1 \%$ and $16.2 \%$, respectively) $\%(\mathrm{P}=$ 0.09). After a median follow-up of 4.4 years and 67 deaths, a survival benefit was not demonstrated (hazard ratio, $0.84 ; 95 \% \mathrm{CI}, 0.52$ to 1.35 ; $\mathrm{P}=0.466$ ). A study by Basi et al. [33] reported similar conclusions.

Currently, the duration of NAC in patients with AGC is inconclusive. In a study of resected AGCs after NAC, physicians established 2 - 4 courses of chemotherapy. If progressive disease (PD) developed after 2 cycles, the patient underwent surgery as soon as possible. If complete remission (CR), partial remission (PR), or stable disease (SD) was achieved, the patient received two additional courses [34].

\subsection{Postoperative Chemotherapy}

Studies have demonstrated that postoperative chemotherapy can reduce the risk of local recurrence and metastasis and improve survival rates [35] [36] [37]. You et al. [35] assessed 126 cases of AGC with R0 resection and D2 lymphadenectomy. Patients were divided into an infusion chemotherapy group (65 cases) and a control group (61 cases) according to postoperative regional infusion of chemotherapy (1000 $\mathrm{mg}$ fluorine and $60 \mathrm{mg}$ cisplatin). No significant differences in the recovery time of intestinal function and the incidence of early and late complications were noted. The 3-year survival and 3-year relapse-free survival rates of patients in the infusion chemotherapy group were significantly increased compared with the control group. Wang et al. [38] observed the effect of RAIC on liver metastasis after gastric cancer resection. They surgically removed T2, N2, and M0 gastric adenocarcinoma without metastasis, and patients were administered RAIC or systemic chemotherapy 30 days after surgery. Both regimens comprised $100 \mathrm{mg} / \mathrm{m}^{2}$ oxaliplatin and $500 \mathrm{mg} / \mathrm{m}^{2}$ fluorodeoxy uridine. Patients who developed liver metastasis during the 3-year follow-up period were offered transarterial chemoembolization. The times to liver metastasis in patients of the RAIC group ( $\mathrm{n}=13)$ and the systemic chemotherapy group $(\mathrm{n}=29)$ were $944 \pm$ 231 days and $506 \pm 77$ days after treatment, respectively. The former was significantly longer than the latter. Their results demonstrated that RAIC is more effective compared with systemic chemotherapy in controlling postoperative liver metastases of gastric cancer.

\section{Response Evaluation}

A therapeutic response evaluation of RAIC is crucial for selecting the next treatment regimen. Medical imaging still plays an important role in response 
evaluation of tumor therapy. The application of functional imaging in the diagnosis and treatment of tumors is becoming increasingly significant [39]-[50].

Survival status is relatively ideal to reflect the efficacy of NAC for gastric cancer [3] [7] [15] [30] [51] [52] [53] [54]. OS was a reliable end-point indicator. Disease-free survival, time to progression, and progression-free survival are main indicators to evaluate efficacy. However, the survival time is recorded after the end of treatment. Its role together with other prognostic indicators of efficacy in the adjustment of the current treatment options and the prediction longterm survival cannot be ignored.

The pathological residue rate of surgically resected specimens after chemotherapy is a relatively objective method to determine the curative effect of NAC. However, in the actual practice of pathological evaluation, it is still difficult to accurately determine the tumor range before chemotherapy and the residual tumor rate.

In addition, biomarkers can also be used as indicators of efficacy [54]-[60].

In short, standards for clinical evaluation of NAC for gastric cancer are lacking. Combining various evaluation indicators may help to improve the accuracy of the evaluation.

\section{Conclusion}

In conclusion, RAIC involves directly delivering chemotherapy drugs to tumor lesions, thereby improving the therapeutic responses and reducing the adverse effects of the drugs. RAIC can effectively reduce the AGC stage and improve the rate of radical resection. Postoperative chemotherapy can reduce the risk of local recurrence and metastasis. Although the number of reports on the use of RAIC for the treatment of AGC is increasing yearly, uniform standards are lacking. In addition, our understanding of important areas about RAIC is limited. These areas include the chemotherapy regimen and doses of chemotherapeutic drugs; the number of courses of preoperative RAIC treatment and the timing of surgical resection; and an evaluation system of treatment response. With the extensive application of RAIC and the development of evidence-based medical research, RAIC, as a treatment method of AGC, will be gradually standardized, and more AGC patients will benefit.

\section{References}

[1] Ferlay, J., Soerjomataram, I., Dikshit, R., Eser, S., Mathers, C., Rebelo, M., Parkin, D.M., Forman, D. and Bray, F. (2015) Cancer Incidence and Mortality Worldwide: Sources, Methods and Major Patterns in GLOBOCAN 2012. International Journal of Cancer, 136, E359-E386. https://doi.org/10.1002/ijc.29210

[2] Mesenas, S., Vu, C., McStay, M., Forshaw, M., Doig, L., Mason, R., Boyle, N. and Meenan, J. (2008) A Large Series, Resection Controlled Study to Assess the Value of Radial EUS in Restaging Gastroesophageal Cancer Following Neoadjuvant Chemotherapy. Diseases of the Esophagus, 21, 37-42. https://doi.org/10.1111/j.1442-2050.2007.00731.x 
[3] He, Q., Li, Y., Ma, L., Ji, X. and Li, G. (2016) Application of FLEEOX Preoperative Chemotherapy via Intra-Arterial and Intravenous Administration in Treatment of Unresectable Locally Advanced Gastric Cancer. Journal of Gastrointestinal Surgery, 20, 1421-1427. https://doi.org/10.1007/s11605-016-3153-8

[4] Li, G.L., Liu, K., Bao, Y., Cao, J.M., Xu, J., Wang, X.L., Wu, B. and Li, J.S. (2012) Retrospective Analysis of 56 Patients with Advanced Gastric Cancer Treated with Combination of Intravenous and Intra-Arterial Intensified Neoadjuvant Chemotherapy. Chinese Medical Journal (English), 125, 780-785.

[5] Li, M., Zhang, J., Wang, D., Zhong, B., Tucker, S., Lu, C., Cheng, J., Cao, C., Xu, J., $\mathrm{Xu}$, J. and Pan, H. (2009) A Phase II Study of Intra-Arterial Chemotherapy of 5-Fluorouracil, Cisplatin, and Mitomycin C for Advanced Nonresectable Gastric Cancer. Anti-Cancer Drugs, 20, 941-945. https://doi.org/10.1097/CAD.0b013e328331af3a

[6] Li, Z.W. and Wang, L. (2013) Progression of Preoperative Regional Arterial Infusion Chemotherapy in Advanced Gastric Cancer. Zhonghua Wei Chang Wai Ke Za Zhi, 16, 197-200.

[7] Wu, Z.F., Cao, Q.H., Wu, X.Y., Chen, C., Xu, Z., Li, W.S., Yao, X.Q. and Liu, F.K. (2015) Regional Arterial Infusion Chemotherapy improves the Pathological Response Rate for Advanced Gastric Cancer with Short-Term Neoadjuvant Chemotherapy. Scientific Reports, 5, 17516. https://doi.org/10.1038/srep17516

[8] Polysalov, V.N., Veriasova, N.N., Dolgikh, S.D. and Gapbarov, A. (2012) Regional Chemotherapy in Locally Advanced and Metastatic Gastric Cancer. Voprosy Onkologii, 58, 762-767.

[9] Iida, T., Hirata, N., Hirakawa, M. and Noguchi, T. (2003) Preoperative Intraarterial Infusion Chemotherapy for Advanced Gastric Cancer-A Retrospective Review of Four Cases. Radiation Medicine, 21, 172-177.

[10] Wu, Z., Zhu, W., Cao, Q., Chen, Z., Wu, X., Chen, C., Xu, Z., Li, W., Yao, X. and Liu, F. (2014) Application of Regional Arterial Infusion Chemotherapy in Short-Term Neoadjuvant Chemotherapy for Advanced Gastric Cancer. Zhonghua Wei Chang Wai Ke Za Zhi, 17, 1092-1095.

[11] Inoue, Y. and Kusunoki, M. (2014) Advances and Directions in Chemotherapy using Implantable Port Systems for Colorectal Cancer: A Historical Review. Surgery Today, 44, 1406-1414. https://doi.org/10.1007/s00595-013-0672-8

[12] Higashi, S., Kato, C., Sakumoto, H., Sumiyoshi, K., Takahahsi, T., Matsumoto, K., Kane, T., Sakoda, M., Takahashi, M., Hosono, Y., Asano, M. and Yamamoto, A. (1999) Arterial Infusion Therapy with Implantable Port for Inoperable Hepatobiliary Tumors. Japanese Journal of Cancer and Chemotherapy, 26, 1764-1767.

[13] Van Cutsem, E., Moiseyenko, V.M., Tjulandin, S., Majlis, A., Constenla, M., Boni, C., Rodrigues, A., Fodor, M., Chao, Y., Voznyi, E., Risse, M.L. and Ajani, J.A. (2006) Phase III Study of Docetaxel and Cisplatin plus Fluorouracil Compared with Cisplatin and Fluorouracil as First-Line Therapy for Advanced Gastric Cancer: A Report of the V325 Study Group. Journal of Clinical Oncology, 24, 4991-4997. https://doi.org/10.1200/JCO.2006.06.8429

[14] Kang, Y.K., Kang, W.K., Shin, D.B., Chen, J., Xiong, J., Wang, J., Lichinitser, M., Guan, Z., Khasanov, R., Zheng, L., Philco-Salas, M., Suarez, T., Santamaria, J., Forster, G. and McCloud, P.I. (2009) Capecitabine/Cisplatin versus 5-fluorouracil/cisplatin as First-Line Therapy in Patients with Advanced Gastric Cancer: A Randomised Phase III Noninferiority Trial. Annals of Oncology, 20, 666-673.

https://doi.org/10.1093/annonc/mdn717 
[15] Wilke, H., Preusser, P., Fink, U., Gunzer, U., Meyer, H.J., Meyer, J., Siewert, J.R., Achterrath, W., Lenaz, L., Knipp, H., et al. (1989) Preoperative Chemotherapy in Locally Advanced and Nonresectable Gastric Cancer: A Phase II Study with Etoposide, Doxorubicin, and Cisplatin. Journal of Clinical Oncology, 7, 1318-1326. https://doi.org/10.1200/JCO.1989.7.9.1318

[16] Chun, E.J. (2017) Ultrasonographic Evaluation of Complications Related to Transfemoral Arterial Procedures. Ultrasonography. https://doi.org/10.14366/usg.17047

[17] Ibrahim, K., Christoph, M., Wunderlich, C., Jellinghaus, S., Loehn, T., Youssef, A., Schoener, L., Quick, S., Mierke, J., Strasser, R.H. and Pfluecke, C. (2017) A Novel Interventional Method for Treating Femoral Pseudoaneurysms: Results from a Monocentric Experience. Eurointervention, 13, 366-370. https://doi.org/10.4244/EIJ-D-16-00865

[18] El-Mawardy, M., Schwarz, B., Landt, M., Sulimov, D., Kebernik, J., Allali, A., Becker, B., Toelg, R., Richardt, G. and Abdel-Wahab, M. (2017) Impact of Femoral Artery Puncture using Digital Subtraction Angiography and Road Mapping on Vascular and Bleeding Complications after Transfemoral Transcatheter Aortic Valve Implantation. Eurointervention, 12, 1667-1673. https://doi.org/10.4244/EIJ-D-15-00412

[19] Panfilov, D.S., Kozlov, B.N., Panfilov, S.D., Kuznetsov, M.S., Nasrashvili, G.G. and Shipulin, V.M. (2016) Puncture Treatment of Pseudoaneurysms of Femoral Arteries with the Use of Human Thrombin. Angiologiya i Sosudistaya Khirurgiya, 22, 62-67.

[20] Morzycki, A., Bhatia, A. and Murphy, K.J. (2017) Adverse Reactions to Contrast Material: A Canadian Update. Canadian Association of Radiologists Journal, 68, 187-193. https://doi.org/10.1016/j.carj.2016.05.006

[21] Caschera, L., Lazzara, A., Piergallini, L., Ricci, D., Tuscano, B. and Vanzulli, A. (2016) Contrast Agents in Diagnostic Imaging: Present and Future. Pharmacological Research, 110, 65-75. https://doi.org/10.1016/j.phrs.2016.04.023

[22] Sikka, A., Bisla, J.K., Rajan, P.V., Chalifoux, L.A., Goodhartz, L.A., Miller, F.H., Yaghmai, V. and Horowitz, J.M. (2016) How to Manage Allergic Reactions to Contrast Agent in Pregnant Patients. American Journal of Roentgenology, 206, 247-252. https://doi.org/10.2214/AJR.15.14976

[23] Beckett, K.R., Moriarity, A.K. and Langer, J.M. (2015) Safe Use of Contrast Media: What the Radiologist Needs to Know. Radiographics, 35, 1738-1750. https://doi.org/10.1148/rg.2015150033

[24] Li, H.Y., Guo, Y.T., Tian, C., Song, C.Q., Mu, Y., Li, Y. and Chen, Y.D. (2017) A Risk Prediction Score Model for Predicting Occurrence of Post-PCI Vasovagal Reflex Syndrome: A Single Center Study in Chinese Population. Journal of Geriatric Cardiology, 14, 509-514.

[25] Frei, E.R. (1982) Multidisciplinary Treatment for Cancer-New Opportunities. International Journal of Radiation Oncology, Biology, Physics, 8, 951-952. https://doi.org/10.1016/0360-3016(82)90108-0

[26] Imano, M., Itoh, T., Satou, T., Sogo, Y., Hirai, H., Kato, H., Yasuda, A., Peng, Y.F., Shinkai, M., Yasuda, T., Imamoto, H., Okuno, K., Shiozaki, H. and Ohyanagi, H. (2010) Prospective Randomized Trial of Short-Term Neoadjuvant Chemotherapy for Advanced Gastric Cancer. European Journal of Surgical Oncology, 36, 963-968. https://doi.org/10.1016/j.ejso.2010.06.012

[27] Li, G.L., Fan, C.G., Bao, Y., Jiang, J., Li, N. and Li, J.S. (2009) Neoadjuvant Chemotherapy of FLEEOX Regimen for Unresectable Gastric Cancer Resulting from Advanced Abdominal Lymph Nodes Metastases. Chinese Journal of Surgery, 47, 1171-1174. 
[28] Dong, X.C., Li, B. and Li, Y.P. (2002) Effect of Preoperative Intra-Arterial Chemotherapy on Apoptosis and p53 Expression of Gastric Cancer. Chinese Journal of Cancer, 21, 1078-1080.

[29] Tao, H.Q. and Zou, S.C. (2002) Effect of Preoperative Regional Artery Chemotherapy on Proliferation and Apoptosis of Gastric Carcinoma Cells. World Journal of Gastroenterology, 8, 451-454. https://doi.org/10.3748/wjg.v8.i3.451

[30] Zhang, C., Li, G., Fan, C., Xu, J., Cao, J., Liu, S. and Li, N. (2012) Comparison of Efficacy of Different Route of Administration of Chemotherapy on Unresectable, Advanced Gastric Cancer. World Journal of Surgical Oncology, 10, 162. https://doi.org/10.1186/1477-7819-10-162

[31] He, Q., Ma, L., Li, Y. and Li, G. (2016) A Pilot Study of an Individualized Comprehensive Treatment for Advanced Gastric Cancer with Para-Aortic Lymph Node Metastasis. BMC Gastroenterology, 16, 8. https://doi.org/10.1186/s12876-016-0422-7

[32] Schuhmacher, C., Gretschel, S., Lordick, F., Reichardt, P., Hohenberger, W., Eisenberger, C.F., Haag, C., Mauer, M.E., Hasan, B., Welch, J., Ott, K., Hoelscher, A., Schneider, P.M., Bechstein, W., Wilke, H., Lutz, M.P., Nordlinger, B., Van Cutsem, E., Siewert, J.R. and Schlag, P.M. (2010) Neoadjuvant Chemotherapy Compared with Surgery Alone for Locally Advanced Cancer of the Stomach and Cardia: European Organisation for Research and Treatment of Cancer Randomized Trial 40954. Journal of Clinical Oncology, 28, 5210-5218. https://doi.org/10.1200/JCO.2009.26.6114

[33] Basi, A., Sohrabkhani, S., Zamani, F., Baghai-Wadji, M., Rabiei, N., Razavi, S.M. and Ajdarkosh, H. (2013) Comparing Efficacy of Preoperative Neo-Adjuvant Chemotherapy and Surgery versus Surgery Alone in Patients with Resectable Gastroesophageal Cancer. International Journal of Hematology-Oncology and Stem Cell Re search, 7, 24-28.

[34] Songun, I., Keizer, H.J., Hermans, J., Klementschitsch, P., de Vries, J.E., Wils, J.A., van der Bijl, J., van Krieken, J.H. and van de Velde, C.J. (1999) Chemotherapy for Operable Gastric Cancer: Results of the Dutch Randomised FAMTX Trial. The Dutch Gastric Cancer Group (DGCG). European Journal of Cancer, 35, 558-562. https://doi.org/10.1016/S0959-8049(98)00429-8

[35] You, X., Qian, H., Qin, L., Wang, Y., Li, W., Lian, Y., Zhao, X., Xu, N., Huang, C., Chen, Z. and Liu, G. (2016) Application Study on Regional Infusion Chemotherapy by Celiac Trunk during Operation in Advanced Gastric Cancer Patients. Chinese Journal of Gastrointestinal Surgery, 19, 1044-1048.

[36] Wu, Y., Wei, Z.W., He, Y.L., Schwarz, R.E., Smith, D.D., Xia, G.K. and Zhang, C.H. (2013) Efficacy of Adjuvant XELOX and FOLFOX6 Chemotherapy after D2 Dissection for Gastric Cancer. World Journal of Gastroenterology, 19, 3309-3315. https://doi.org/10.3748/wjg.v19.i21.3309

[37] Wu, Z., Ma, S., Jing, S., Deng, Q., Zheng, Z., Wu, K., Li, J., Chen, S., Tang, R. and Li, X. (2014) Effect of Hyperthermic Intraperitoneal Perfusion Chemotherapy in Combination with Intravenous Chemotherapy as Postoperative Adjuvant Therapy for Advanced Gastric Cancer. Hepatogastroenterology, 61, 972-977.

[38] Wang, Y.Y., Zhang, W., Qian, S., Liu, R., Kan, Z.X. and Wang, J.H. (2012) The Effect of Locoregional Transarterial Infusion Chemotherapy on Liver Metastasis after Gastric Cancer Resection. Journal of International Medical Research, 40, 1141-1148. https://doi.org/10.1177/147323001204000334 
[39] Yang, K., Zhang, X.M., Yang, L., Xu, H. and Peng, J. (2016) Advanced Imaging Techniques in the Therapeutic Response of Transarterial Chemoembolization for Hepatocellular Carcinoma. World Journal of Gastroenterology, 22, 4835-4847. https://doi.org/10.3748/wjg.v22.i20.4835

[40] Yang, L., Zhang, X.M., Tan, B.X., Liu, M., Dong, G.L. and Zhai, Z.H. (2012) Computed Tomographic Perfusion Imaging for the Therapeutic Response of Chemoembolization for Hepatocellular Carcinoma. Journal of Computer Assisted Tomography, 36, 226-230. https://doi.org/10.1097/RCT.0b013e318245c23c

[41] Yang, L., Zhang, X.M., Zhou, X.P., Tang, W., Guan, Y.S., Zhai, Z.H. and Dong, G.L. (2010) Correlation between Tumor Perfusion and Lipiodol Deposition in Hepatocellular Carcinoma after Transarterial Chemoembolization. Journal of Vascular and Interventional Radiology, 21, 1841-1846. https://doi.org/10.1016/j.jvir.2010.08.015

[42] Hoink, A.J., Schulke, C., Koch, R., Lohnert, A., Kammerer, S., Fortkamp, R., Heindel, W. and Buerke, B. (2017) Response Evaluation of Malignant Liver Lesions after TACE/SIRT: Comparison of Manual and Semi-Automatic Measurement of Different Response Criteria in Multislice CT. Rofo, 189, 1067-1075. https://doi.org/10.1055/s-0043-116220

[43] Ludwig, J.M., Camacho, J.C., Kokabi, N., Xing, M. and Kim, H.S. (2015) The Role of Diffusion-Weighted Imaging (DWI) in Locoregional Therapy Outcome Prediction and Response Assessment for Hepatocellular Carcinoma (HCC): The New Era of Functional Imaging Biomarkers. Diagnostics (Basel), 5, 546-563. https://doi.org/10.3390/diagnostics5040546

[44] Hussain, H.K., Barr, D.C. and Wald, C. (2014) Imaging Techniques for the Diagnosis of Hepatocellular Carcinoma and the Evaluation of Response to Treatment. Seminars in Liver Disease, 34, 398-414. https://doi.org/10.1055/s-0034-1394140

[45] Yuan, Z., Li, W.T., Ye, X.D., Zhu, H.Y. and Peng, W.J. (2014) Novel Functional Magnetic Resonance Imaging Biomarkers for Assessing Response to Therapy in Hepatocellular Carcinoma. Clinical and Translational Oncology, 16, 599-605. https://doi.org/10.1007/s12094-013-1147-5

[46] Jiang, T., Zhu, A.X. and Sahani, D.V. (2013) Established and Novel Imaging Biomarkers for Assessing Response to Therapy in Hepatocellular Carcinoma. Journal of Hepatology, 58, 169-177. https://doi.org/10.1016/j.jhep.2012.08.022

[47] De Raffele, E., Mirarchi, M., Cuicchi, D., Lecce, F. and Cola, B. (2017) Evolving Role of FDG-PET/CT in Prognostic Evaluation of Resectable Gastric Cancer. World Journal of Gastroenterology, 23, 6923-6926. https://doi.org/10.3748/wjg.v23.i38.6923

[48] Cheng, J., Wang, Y., Zhang, C.F., Wang, H., Wu, W.Z., Pan, F., Hong, N. and Deng, J. (2017) Chemotherapy Response Evaluation in a Mouse Model of Gastric Cancer using Intravoxel Incoherent Motion Diffusion-Weighted MRI and Histopathology. World Journal of Gastroenterology, 23, 1990-2001. https://doi.org/10.3748/wjg.v23.i11.1990

[49] Yuan, J., Lo, G. and King, A.D. (2016) Functional Magnetic Resonance Imaging Techniques and Their Development for Radiation Therapy Planning and Monitoring in the Head and Neck Cancers. Quantitative Imaging in Medicine and Surgery, 6, 430-448. https://doi.org/10.21037/qims.2016.06.11

[50] Bozgeyik, Z., Onur, M.R. and Poyraz, A.K. (2013) The Role of Diffusion Weighted Magnetic Resonance Imaging in Oncologic Settings. Quantitative Imaging in Medicine and Surgery, 3, 269-278. 
[51] He, Q., Li, G., Ji, X., Ma, L., Wang, X., Li, Y. and Fan, C. (2017) Impact of the Immune Cell Population in Peripheral Blood on Response and Survival in Patients Receiving Neoadjuvant Chemotherapy for Advanced Gastric Cancer. Tumor Biology, 39, 1-8. https://doi.org/10.1177/1010428317697571

[52] Tahara, T., Shibata, T., Okubo, M., Kawamura, T., Horiguchi, N., Yoshida, D., Ishizuka, T., Nagasaka, M., Nakagawa, Y. and Ohmiya, N. (2017) Evaluations of Primary Lesions by Endoscopy Clearly Distinguishes Prognosis in Patients with Gastric Cancer Who Receive Chemotherapy. PLoS ONE, 12, e173663.

https://doi.org/10.1371/journal.pone.0173663

[53] Kim, J.S., Kang, S.H., Moon, H.S., Sung, J.K., Jeong, H.Y. and Sul, J.Y. (2016) Clinical Outcome of Doublet and Triplet Neoadjuvant Chemotherapy for Locally Advanced Gastric Cancer. The Korean Journal of Gastroenterology, 68, 245-252. https://doi.org/10.4166/kjg.2016.68.5.245

[54] Qu, J. and Qu, X. (2016) The Predictors of Response to Neoadjuvant Chemotherapy in Patients with Locally Advanced Gastric Cancer. Cancer Biomarkers, 17, 49-54. https://doi.org/10.3233/CBM-160616

[55] Liu, K., Min, X.L., Peng, J., Yang, K., Yang, L. and Zhang, X.M. (2016) The Changes of HIF-1alpha and VEGF Expression after TACE in Patients with Hepatocellular Carcinoma. Journal of Clinical Medicine Research, 8, 297-302. https://doi.org/10.14740/jocmr2496w

[56] Mamdouh, S., Khorshed, F., Aboushousha, T., Hamdy, H., Diab, A., Seleem, M. and Saber, M. (2017) Evaluation of Mir-224, Mir-215 and Mir-143 as Serum Biomarkers for HCV Associated Hepatocellular Carcinoma. Asian Pacific Journal of Cancer Prevention, 18, 3167-3171.

[57] Fornaro, L., Vivaldi, C., Lin, D., Xue, H., Falcone, A., Wang, Y., Crea, F. and Bootman, M.D. (2017) Prognostic Relevance of a T-Type Calcium Channels Gene Signature in Solid Tumours: A Correlation Ready for Clinical Validation. PLoS ONE, 12, e182818. https://doi.org/10.1371/journal.pone.0182818

[58] Mahfouz, N., Tahtouh, R., Alaaeddine, N., El, H.J., Sarkis, R., Hachem, R., Raad, I. and Hilal, G. (2017) Gastrointestinal Cancer Cells Treatment with Bevacizumab Activates a VEGF Autoregulatory Mechanism Involving Telomerase Catalytic Subunit hTERT via PI3K-AKT, HIF-1alpha and VEGF Receptors. PLoS ONE, 12, e179202. https://doi.org/10.1371/journal.pone.0179202

[59] Liu, K., Yang, L., Zhang, X.M., Zhou, Y., Zhu, T., Miao, N.D., Ren, Y.J., Xu, H., Min, X.L., Peng, J., Yang, K. and Yang, S. (2017) HIF-1 $\alpha$ and VEGF Levels for Monitoring Hepatocellular Carcinoma Treatment Response to Transcatheter Arterial Chemoembolization. Translational Cancer Research, 6, 1043-1049. https://doi.org/10.21037/tcr.2017.08.32

[60] Min, X.L., Yang, L., Zhang, X.M., Zhou, Y., Miao, N.D., Ren, Y.J., Xu, H., Liu, K., Peng, J. and Yang, K. (2017) Changes of Tryptase in Patients with Hepatocellular Carcinoma after Transarterial Chemoembolization. Translational Cancer Research, 6, 1061-1067. https://doi.org/10.21037/tcr.2017.08.43 